\section{\$32. Reducing Thermal Conductivity of Thermoelectric Materials by Using a Narrow Wire Geometry}

Hasegawa, Y. (Saitama Univ.), Nakamura, H., Sato, M.

Dependence of thermal conductivity of narrow wires made from bismuth and covalently bonded materials on wire diameter was numerically calculated by considering contributions of mean free paths of carriers and phonons. The results suggest that a reduction in the thermal conductivity should be observable in a bismuth wire having a diameter of less than $1 \mu \mathrm{m}$ sample. A reduction of nearly $20 \%$ in the temperature range of $150 \mathrm{~K}$ to $300 \mathrm{~K}$ is expected due to using a narrow wire geometry. Such a geometry reduces mobility and the thermal conductivity of the carriers, which is the dominant component, while the thermal conductivity due to phonons was dramatically reduced by using narrow wires at temperatures under $50 \mathrm{~K}$ due to the longer mean free path phonons. The thermal conductivity of materials with covalent bonding such as silicon was also estimated, and it is expected that the thermal conductivity of silicon wire could be reduced due to the mean free path of phonons being longer than that of the carriers. The results suggest that it should be possible to enhance the figure of merit by reducing the thermal conductivity through using wire geometries having diameters of less than $100 \mathrm{~nm}$ in materials having low mobilities, high thermal conductivities and high Debye temperatures.

We calculated the dependence of the thermal conductivity on wire diameter for bismuth and silicon, which is typical covalently bonded materials, showing Figs. 1 and 2. In bismuth, the thermal conductivity due to the carriers was mainly reduced at temperatures over $150 \mathrm{~K}$, while the thermal conductivity due phonons was dramatically reduced below $20 \mathrm{~K}$ in wires having diameters less than $1 \mu \mathrm{m}$. The reduction in the thermal conductivity is strongly related to relationship between the mean free paths of the carriers and phonons. The thermal conductivity and mobility of bismuth were simultaneously reduced by using a narrow wire geometry since the mean free path of the carriers was much longer than that of phonons. Therefore, it is generally not effective to use a narrow wire geometry to reduce the thermal conductivity of materials that have high mobilities and low thermal conductivities (e.g., bismuth). On the other hand, for bulk materials with high thermal conductivities, low mobilities and high Debye temperatures (e.g., silicon), it is possible to reduce the thermal conductivity by using a wire geometry while maintaining the power factor provided that they can be formed into nanoscale structures. By using a wire or thin film geometry, it is possible to reduce the thermal conductivity and enhance the figure of merit of thermoelectric materials without using the quantum effect when the wire diameter or thickness of the thin film is optimized (typically less than $100 \mathrm{~nm}$ ). A nanotechnology that makes it possible to reduce the length so that it is less than the mean free path of phonons will open a new phase in the development for thermoelectric materials with high power factors and high thermal conductivities.

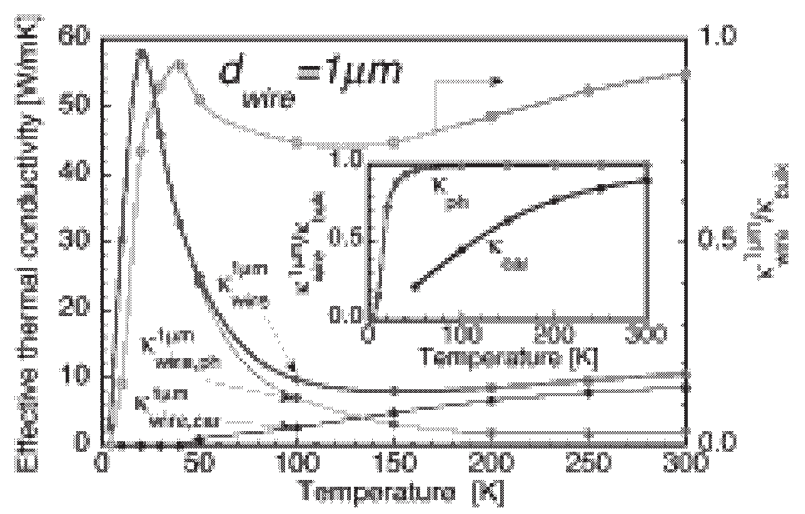

Fig. 1. Temperature dependence of the effective thermal conductivity in a 1-mm-diameter bismuth wire and the reduction ratio $\kappa_{\text {wire }}^{1 \mu m} / \kappa_{\text {bulk }}$ relative to bulk bismuth. $\boldsymbol{\kappa}_{\text {wire,car }}^{1 \mu m}$ and $\boldsymbol{\kappa}_{\text {wire,ph }}^{1 \mu m}$ represent the contributions to the total thermal conductivity $\kappa_{\text {wire }}^{1 \mu m}$ from the carriers and phonons, respectively. The inset shows the reduction ratio due to the carriers $\kappa_{c a r}$ and phonons $\kappa_{p h}$ for a $1-\mu \mathrm{m}$-diameter wire.

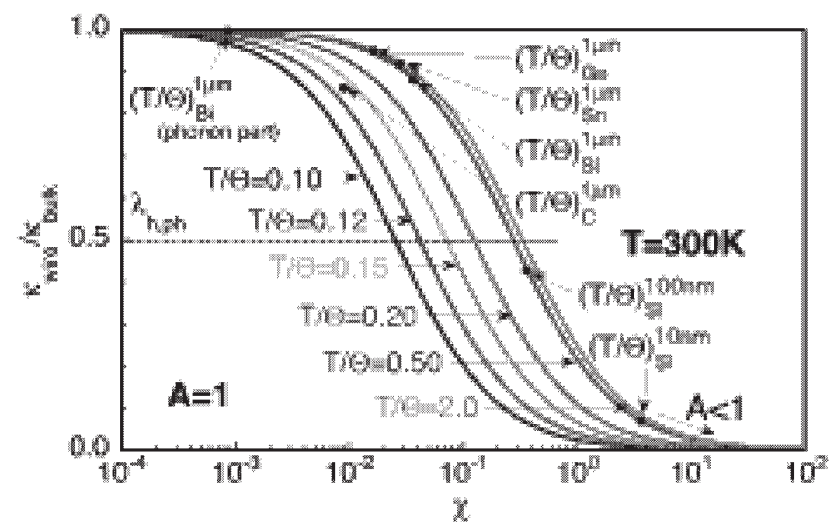

Fig. 2. $\chi$ dependence of the normalized thermal conductivity $\kappa_{\text {wire }} / \kappa_{\text {bulk }}$ at various normalized Debye temperatures $\frac{\Theta}{T}$ within covalent bonded materials (Si, Ge, C and $\mathrm{Sn}$ ) and bismuth at $300 \mathrm{~K}$. The label $\left(\frac{T}{\Theta}\right)_{M}^{1 \mu m}$ indicates the normalized Debye temperature for a $1-\mu \mathrm{m}$-diameter wire for material $\mathrm{M}$.

1) Masayuki Murata, Daiki Nakamura, Yasuhiro Hasegawa, Takashi Komine, Takashi Taguchi and Shinichiro Nakamura, Christopher M. Jaworski, Vladimir Jovovic and Joseph P. Heremans, "Mean free path limitation of thermoelectric properties on bismuth nano-wire", Journal of Applied Physics, 105, 113706 19 (2009) 